\title{
Cancer and cholesterol: understanding the V-shaped association in patients with diabetes
}

\author{
Eric L. Ding ScD, Frank B. Hu MD PhD
}

$\infty \quad$ See related research paper by Yang and colleagues, page 427

$\mathrm{E}$ levated serum glucose and cholesterol levels have been widely shown to be linked to increased cardiovascular mortality and all-cause mortality. Hypercholesterolemia is also a well-established risk factor for cardiovascular disease among patients with type 2 diabetes. The association between high cholesterol levels and increased risk of cancer has been a long-standing area of interest and investigation because the pathway for cholesterol synthesis may produce various tumorigenic compounds and because cholesterol serves as a precursor for the synthesis of many sex hormones linked to increased risk of various cancers. However, disease prediction based on biological hypotheses often is not well reflected in actual clinical risk. Such complexity is underscored by divergent findings from epidemiologic studies on serum cholesterol levels and cancer risk.

In this issue of $C M A J$, Yang and colleagues ${ }^{1}$ conducted a prospective study of the association between low-density lipoprotein (LDL) cholesterol levels and risk of cancer among Chinese patients with type 2 diabetes. Results indicated a consistent and striking $\mathrm{V}$-shaped association. The lowest risk of cancer was observed among those with an intermediate LDL cholesterol level $(\geq 2.80$ to $<3.80 \mathrm{mmol} / \mathrm{L})$. About a $50 \%$ higher risk of cancer was observed among patients with an LDL cholesterol level either above or below that range. Overall, the findings were robust in both crude and adjusted models, and results were consistent among users and nonusers of statins. A V-shaped association was also observed between LDL cholesterol and all-cause mortality.

What warrants careful consideration is whether the association is biologically causal or merely confounded by other risk factors, including socioeconomic status. A V-shaped association between LDL cholesterol and cancer risk suggests that multiple mechanisms are involved. LDL cholesterol is unlikely the sole or direct causal factor.

More than a decade ago, Jacobs and colleagues ${ }^{2}$ observed that low total cholesterol levels were associated with an increased risk of cancer. However, this finding was considered to be attributable to confounding or reverse causation by subclinical cancer. Low cholesterol levels are commonly observed in people who are ill (e.g., patients with cancer) and those with unhealthy lifestyle characteristics, such as smoking and heavy drinking. ${ }^{3}$ Furthermore, 3 meta-analyses of randomized controlled trials showed that the use of statins, which inhibits cholesterol synthesis, was not associated with increased or decreased risk of overall or major cancers. ${ }^{4-6}$ In addition, results of a cohort study of total cholesterol by Wannamethee and colleagues ${ }^{3}$ indicated that the increased risk of cancer was mostly concentrated in the early years of

\section{Key points}

- Disease prediction based on biological hypotheses often is not well reflected in actual clinical risk.

- We must carefully consider whether the association between low levels of low-density lipoprotein (LDL) cholesterol is biologically causal or merely confounded by other risk factors, including socioeconomic status.

- The true clinical utility of LDL cholesterol in risk stratification of a variety of cancers remains unclear.

- Further investigations are required about the implications for statin therapy and the ability of LDL cholesterol to predict the development of specific cancers in the general population as well as in patients with diabetes.

follow-up and that the relation attenuated after excluding these early years. This finding suggests reverse causation that preclinical cancer led to a drop in cholesterol.

On the other hand, a meta-analysis of data from placebocontrolled statin trials showed that, although the incremental absolute reduction in LDL cholesterol did not correlate with cancer risk, achieving low levels of LDL cholesterol was associated with an increased risk of cancer. ${ }^{7}$ Furthermore, results of the studies by Jacobs and colleagues ${ }^{2}$ and by Yang and colleagues ${ }^{1}$ showed little attenuation of the positive association between low LDL cholesterol levels and risk of cancer after the exclusion of data for patients who died in the early years of follow-up. Thus, the relation between low levels of LDL cholesterol and cancer remains controversial, and its true mechanism remains elusive.

If proven to be true, the relation between low LDL cholesterol levels and increased risk of cancer may be relevant for clinical practice, given that current recommendations for the treatment of LDL cholesterol, which assume that lower is always better, advocate greater intensity of interventions to push LDL cholesterol target levels ever lower. In the absence of definitive biological mechanisms confirming that low LDL cholesterol levels increase the risk of cancer and given the results of randomized trials indicating no increased risk of cancer with the use of statins, lowering LDL cholesterol still remains a top priority in the treatment of hypercholesterolemia.

The positive association observed by Yang and colleagues between high levels of LDL cholesterol and risk of cancer appears to be more biologically plausible, although the possi-

Eric Ding and Frank Hu are with the Department of Nutrition, and Frank Hu is with the Department of Epidemiology, Harvard School of Public Health, Boston, Mass. Frank Hu is also with the Department of Medicine, Brigham and Women's Hospital and Harvard Medical School, Boston, Mass. 
bility of confounding remains. Besides serving as a potential indicator of production of tumorigenic compounds from cholesterol synthesis, ${ }^{1}$ cholesterol is an important biological precursor to many sex steroid hormones such as estrogen, which has been implicated in several cancers in women. ${ }^{8,9}$ Nevertheless, LDL cholesterol levels generally have not been strongly associated with circulating estrogens in most studies, ${ }^{10}$ and epidemiologic studies on the relation between elevated plasma estrogen levels and cancer risk have not always been concordant with findings from clinical trials of estrogen therapy. However, all of these mechanisms are indirect and unlikely to explain completely the observed positive association between high LDL cholesterol levels and cancer risk.

Alternatively, confounding by indication for the use of statins, lifestyle factors or socioeconomic status may explain the positive association between high levels of LDL cholesterol and cancer risk. It has been widely shown that socioeconomic gradients exist in the incidence of cancer and in the use of and adherence to statin treatments. ${ }^{11-13}$ Yang and colleagues observed much disparity in access to care and drugs in Hong Kong by socioeconomic status. ${ }^{1}$ They observed stronger associations between high levels of LDL cholesterol and cancer among patients who did not take statins than among statin users. Their findings suggest that indication for the use of statins and adherence are unlikely to explain the observed association. However, because the study did not comprehensively adjust for many other major risk factors for cancer or conduct a risk-prediction analysis, the true clinical utility of LDL cholesterol in risk stratification of a variety of cancers remains unclear.

Overall, the study by Yang and colleagues raises many questions about the aggressive use of statins, cholesterol-mediated biological mechanisms and the potential clinical utility of LDL cholesterol to predict cancer risk. However, further investigations are required about the implications of cholesterol-lowering drug therapy on cancer risk and the ability of LDL cholesterol to predict the development of specific cancers in the general population as well as in patients with diabetes.
Competing interests: None declared.

Contributors: Both of the authors contributed to the content of this article, revised it critically and approved the final version for publication.

Acknowledgements: Eric Ding is supported by a postdoctoral fellowship award from the American Diabetes Association. Frank Hu is supported by US National Institutes of Health grants DK58845 and HL60712.

\section{REFERENCES}

1. Yang X, So W, Ko GTC, et al. Independent associations between low-density lipoprotein cholesterol and cancer among patients with type 2 diabetes mellitus. CMAJ 2008;179:427-37.

2. Jacobs D, Blackburn H, Higgins M, et al. Report of the Conference on Low Blood Cholesterol: mortality associations. Circulation 1992;86:1046-60.

3. Wannamethee G, Shaper AG, Whincup PH, et al. Low serum total cholesterol concentrations and mortality in middle aged British men. BMJ 1995;311:409-13.

4. Bonovas S, Filioussi K, Tsavaris N, et al. Statins and cancer risk: a literature-based meta-analysis and meta-regression analysis of 35 randomized controlled trials. $J$ Clin Oncol 2006;24:4808-17.

5. Dale KM, Coleman CI, Henyan NN, et al. Statins and cancer risk: a meta-analysis. JAMA 2006;295:74-80.

6. Browning DR, Martin RM. Statins and risk of cancer: a systematic review and meta-analysis. Int J Cancer 2007;120:833-43.

7. Alsheikh-Ali AA, Maddukuri PV, Han H, et al. Effect of the magnitude of lipid lowering on risk of elevated liver enzymes, rhabdomyolysis, and cancer: insights from large randomized statin trials. J Am Coll Cardiol 2007;50:409-18.

8. Anderson GL, Limacher M, Assaf AR, et al. Effects of conjugated equine estrogen in postmenopausal women with hysterectomy: the Women's Health Initiative randomized controlled trial. JAMA 2004;291:1701-12.

9. Rossouw JE, Anderson GL, Prentice RL, et al. Risks and benefits of estrogen plus progestin in healthy postmenopausal women: principal results from the Women's Health Initiative randomized controlled trial. JAMA 2002;288:321-33.

10. Lamon-Fava S, Barnett JB, Woods MN, et al. Differences in serum sex hormone and plasma lipid levels in Caucasian and African-American premenopausal women. J Clin Endocrinol Metab 2005;90:4516-20.

11. Thomsen RW, Johnsen SP, Olesen AV, et al. Socioeconomic gradient in use of statins among Danish patients: population-based cross-sectional study. Br J Clin Pharmacol 2005;60:534-42.

12. Odubanjo E, Bennett K, Feely J. Influence of socioeconomic status on the quality of prescribing in the elderly - a population based study. Br J Clin Pharmacol 2004;58:496-502.

13. Cheng CW, Woo KS, Chan JC, et al. Association between adherence to statin therapy and lipid control in Hong Kong Chinese patients at high risk of coronary heart disease. Br J Clin Pharmacol 2004;58:528-35.

Correspondence to: Dr. Frank B. Hu, Harvard School of Public Health, Department of Nutrition, 655 Huntington Ave., Boston MA 02115 USA; fax 617 432-2435; frank.hu@ channing.harvard.edu 